\title{
THE THERAPEUTIC APPROACH OF SOFT - TISSUE SARCOMAS. A 12 CASES ANALYSIS
}

\author{
doi: 10.2478/rojost-2018-0069 \\ V. Georgeanu ${ }^{1,2}$, T. Atasiei ${ }^{1}$, D. Gartonea ${ }^{1}$, B. Shazam ${ }^{1}$, G. Goleșteanu ${ }^{1}$, Șt. Cristea ${ }^{1,2}$ \\ ${ }^{1}$ St. Pantelimon Hospital, Bucharest \\ 2 "Carol Davila" University of Medicine and Pharmacy, Bucharest, Romania
}

Introduction. Soft Tissue Sarcomas (STS) is a group of rare malignant tumors with mesenchymal tissue origin. At present, over 50 histopathological types with typical chromosome changes are described. Treatment is multidisciplinary, centered on the surgical approach.

Method. Between 2014 and 2016, 12 STS cases were treated in our clinic: 3 liposarcomas, 2 synovial sarcomas, 1 angiosarcoma, 2 rhabdomyosarcomas, 2 myxofibrosarcomas, 1 fibroblast sarcoma and 1 clear cell sarcoma. With the exception of angiosarcoma that benefited only from chemotherapy, the other cases were surgically approached, followed by radiotherapy in 7 cases, and chemotherapy in one case.

Results. Patient follow-up was for a minimum of 2 years. In 3 cases (fibroblast sarcoma, clear-cell sarcoma, synovial sarcoma), a local recurrence occurred between 3 and 9 months. In 2 cases (fibroblastic sarcoma, synovial sarcoma), pulmonary metastases occurred between 6 and 18 months and the patients died at 8 months and 2 years respectively. The rest of the cases were clinically and imagistically assessed (local MRI, lung CT) at 6 months for at least 2 years and showed no signs of local recurrence or metastasis.

Conclusions. The evolution of STS depends on the histological type and to the stage of diagnosis (local extension, degree of differentiation and presence of metastases). Therapeutic approach should be centered on an accurate resection, within the limits of oncological safety even with re-resection. Postoperative radiotherapy is used in most of the cases, and chemotherapy is reserved for special cases with unfavorable local evolution or metastasis.

Keywords: Soft Tissue Sarcoma, aggressive tumors, multidisciplinary approach 